\title{
Hypertrophie osseuse buccale chez l'édenté complet : une fatalité ò contourner !
}

\section{Oral bone hypertrophy in full toothless: inevitable to circumvent!}

\section{Résumé}

\section{MOTS-CLEFS :}

- Hypertrophie osseuse, exostose, torus, espace prothétique, aménagement chirurgical

\section{KEYWORDS:}

- Bone hypertrophy, exostosis, torus, prosthetic space, surgical management

$\operatorname{AOS} n^{\circ} 275-2016$
En prothèse amovible complète, les réhabilitations prothétiques sont parfois difficiles, surtout en présence d'un environnement ostéomuqueux défavorable, empêchant la mise en place de la prothèse.

En effet, une hypertrophie crestale et tubérositaire importantes impose un aménagement chirurgical de l'espace prothétique utile, car ces obstacles anatomiques contraignent le praticien à des compromis néfastes pour la stabilité des prothèses, tant au niveau de l'étendue des bases qu'au niveau de l'occlusion et de l'esthétique.

À travers ce travail, nous allons essayer d'illustrer les difficultés inhérentes à l'insuffisance de l'espace prothétique dans le sens horizontal et de proposer une conduite à tenir adéquate face à ce problème pour un équilibre prothétique optimal.
Abstract

In removable full prosthesis, prosthetic rehabilitation can be difficult, especially in the presence of an unfavorable ostéomuqueux environment, preventing the establishment of the prosthesis.

Indeed, a significant hypertrophy and crestal tuberosity requires surgical planning of the prosthetic space useful because these anatomical obstacles forcing the practitioner to harmful to compromise the stability of the prostheses, in terms of the extent of the base at the level occlusion and aesthetics.

Through this work, we will try to illustrate the difficulties inherent in the lack of prosthetic space in the horizontal direction and propose a course of action adequately address this problem for optimal prosthetic balance.

\begin{tabular}{|l}
\hline Anissa REGRAGUI', professeur assistante en Prothèse Amovible \\
Amal SEFRIOUI, professeur agrégée en Prothèse Amovible \\
Nadia MERZOUK, professeur d'enseignement supérieur en Prothèse Amovible \\
et chef du service de Prothèse Amovible au centre de consultation et des traitements \\
dentaires de Rabat-CCTD \\
Salwa BERRADA², professeur d'enseignement supérieur \\
'Lot. Al Wifakia; Imm. 89; Entrée B; Apt 14; TEMARA-MAROC. \\
anissaregragui@yahoo.fr \\
(212) G70057685 \\
'Université Mohamed V, Faculté de médecine dentaire de Rabat, Avenue Allal El Fassi, \\
Rue Mohammed Jazouli, Madinat Al Irfane, BP 6212, Rabat - Instituts, Maroc
\end{tabular}




\section{INTRODUCTION}

La thérapeutique de l'édentement complet ou partiel de grande étendue, implique une exploitation maximale des zones anatomiques susceptibles d'optimiser la sustentation et la rétention prothétiques. Ces impératifs fondamentaux de la prothèse adjointe se heurtent parfois à des contraintes qui les rendent difficiles, voire impossibles, à réaliser.

La présence d'hypertrophies osseuses au niveau des zones support de la prothèse (crête édentée, tubérosité maxillaire ou palais) peut compromettre la qualité de la réalisation prothétique, ce qui imposera un aménagement chirurgical de l'espace prothétique utile car ces obstacles anatomiques peuvent contraindre le praticien à des compromis néfastes pour la stabilité des prothèses tant au niveau de l'étendue des bases qu'au niveau de l'occlusion et de l'esthétique. [1] [2]

En effet, le tissu osseux peut présenter une hypertrophie lorsqu'il subit une augmentation de volume; on distingue l'hypertrophie généralisée et localisée; l'exostose est une hypertrophie localisée de l'os corticale du maxillaire et de la mandibule. Elle se trouve généralement le long des alvéoles ou sur le palais dur et, en fonction de sa localisation et de son étendue, elle peut être classée comme torus mandibulaire, torus maxillaire ou exostose maxillaire vestibulaire ou linguale. [3] [4]

Les exostoses maxillaires se rencontrent le plus souvent à côté des molaires, s'étendant parfois jusqu'à la $2^{\mathrm{e}}$ prémolaire et, dans de rares cas, à la canine et aux incisives.

Variables dans l'expression, les exostoses maxillaires peuvent être lisses et continues avec la crête, ou se manifester sous forme de nodules uniques ou multiples plus ou moins discrètes.

Au niveau du maxillaire, elles sont le plus souvent vestibulaires, par contre à la mandibule, leur localisation est souvent linguale. [5] [6]

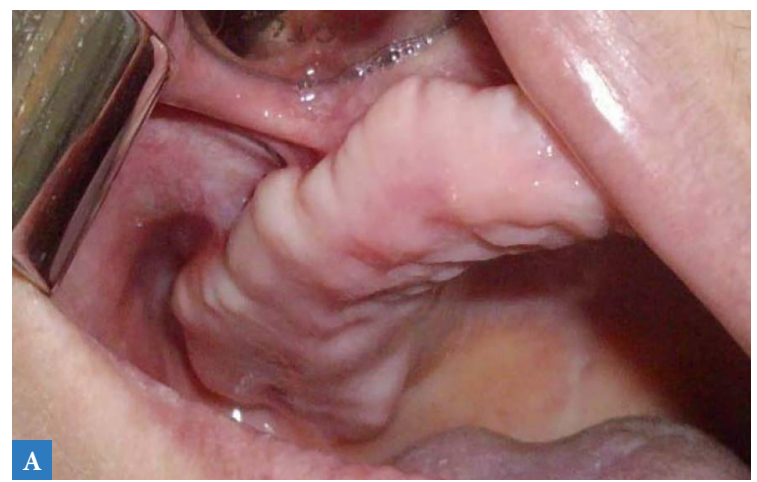

\section{OBSERVATION CLINIQUE}

Nous illustrons, à travers un cas clinique, les difficultés inhérentes à l'hypertrophie osseuse dans le sens horizontal et nous proposons une conduite à tenir adéquate pour un équilibre prothétique optimal.

\section{État initial}

Il s'agit d'une patiente âgée de 46 ans, apparemment en bon état de santé générale, originaire du sud-ouest du Maroc, non appareillée malgré l'ancienneté de son édentement. Ceci est dû à l'insuffisance horizontale de l'espace prothétique disponible interférant avec les organes para-prothétiques (joues et lèvres) et rendant impossible la mise en place prothétique.

L'examen clinique révèle un édentement complet au maxillaire et un édentement partiel de classe III de Kennedy-Applegate à la mandibule.

$\mathrm{Au}$ maxillaire, les crêtes sont hypertrophiées dans le sens horizontal surtout au niveau des régions prémolaires-molaires droites. Les tubérosités sont volumineuses présentant des invaginations osseuses irrégulières et des contre-dépouilles vestibulaires importantes. La fibromuqueuse est ferme et adhérente aux plans profonds. (Fig. 1a et b)

À la mandibule, la crête édentée présente une exostose osseuse recouverte d'une fibromuqueuse ferme et adhérente. (Fig. 2)

L'examen radiologique tomodensitométrique met en évidence des bases osseuses hypertrophiées et de densité importante. Il informe sur la présence d'une proximité sinusienne et une proximité du canal palatin et permet d'estimer l'épaisseur de la fibromuqueuse en regard des tubérosités. (Fig. 3a, b et c)

L'examen biologique a montré une baisse du taux de la vitamine $\mathrm{D}$.

\section{Étude préprothétique}

Toute approche chirurgicale importante nécessite une analyse soigneuse du cas clinique pour une évaluation

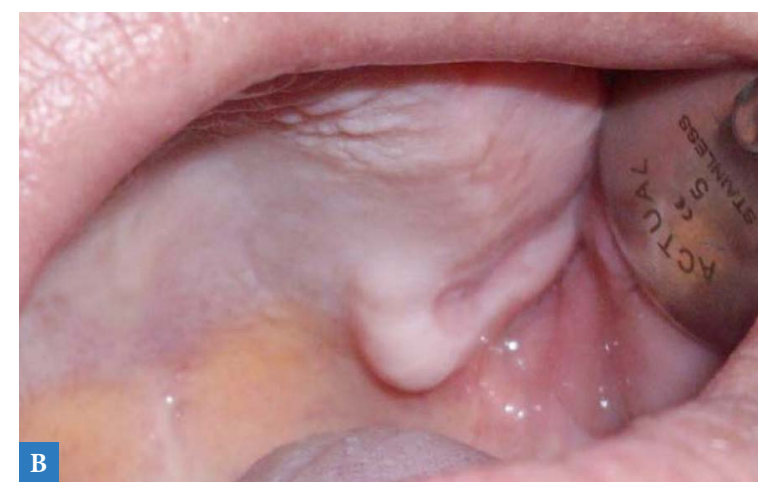

$\triangle$ Fig. 1a et b : Hypertrophie osseuse maxillaire généralisée horizontale interférant avec les organes para-prothétiques (joues et lèvres). 


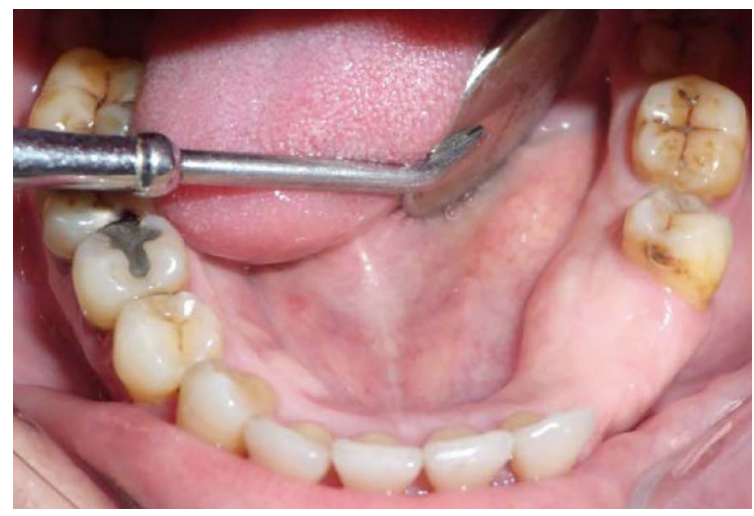

$\triangle$ Fig. 2 : Hypertrophie osseuse mandibulaire localisée au secteur édenté.

précise de l'étendue et de la quantité de tissus à supprimer, car la qualité du résultat dépend de l'exactitude du diagnostic. L'étude des modèles d'étude permet : I une appréciation de l'espace prothétique disponible ; \l'évaluation des aménagements chirurgicaux à apporter ;

Ila réalisation des prothèses transitoires après rectification des modèles.

Le transfert des modèles d'étude sur articulateur par le biais de maquettes d'occlusion se fait après enregistrement du rapport maxillo-mandibulaire en relation centrée et de la dimension verticale physiologique. Il confirme une hypertrophie importante des crêtes maxillaires dans le sens horizontal, l'espace prothétique disponible paraît suffisant en hauteur (Fig. 4a et b).

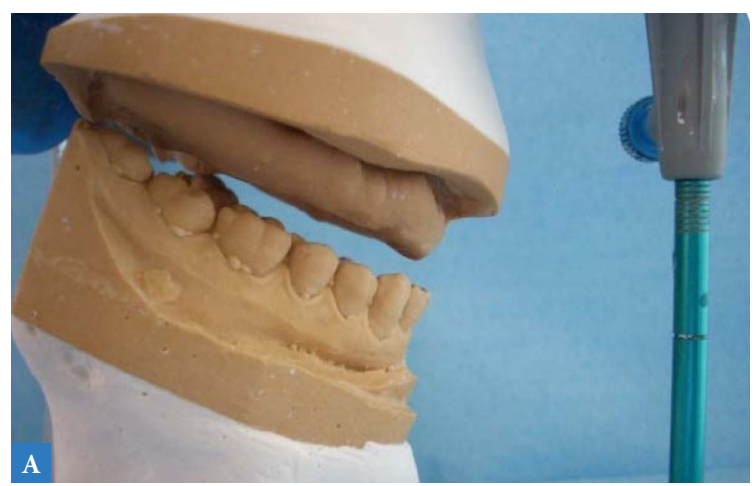

$\triangle$ Fig. 4a et b : Espace prothétique disponible suffisant en hauteur.

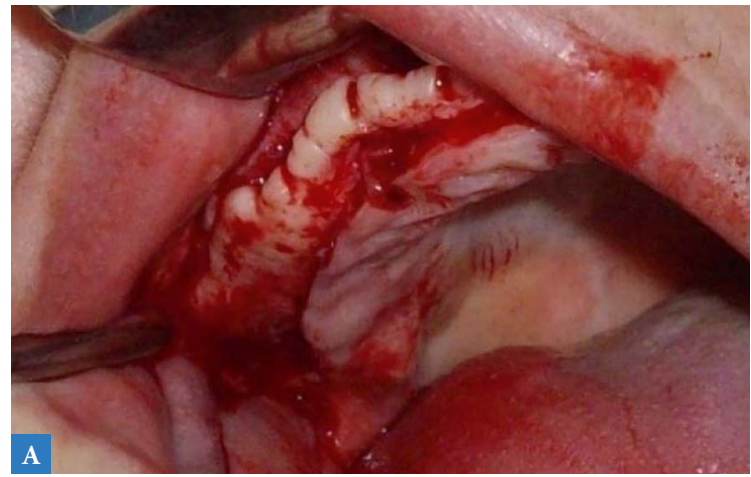

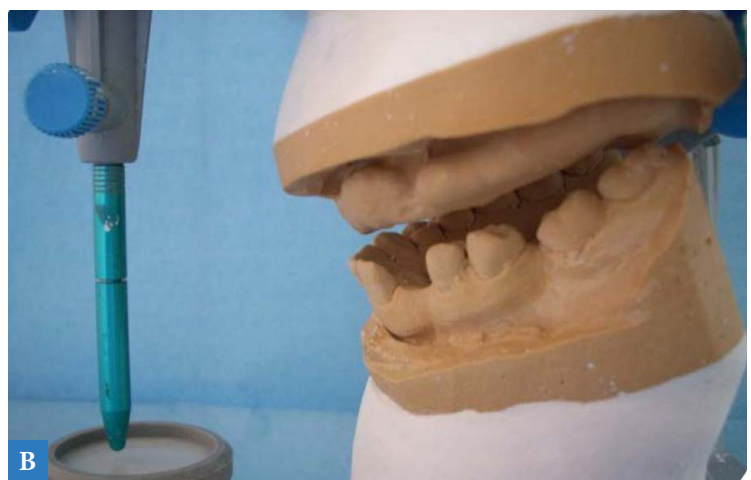
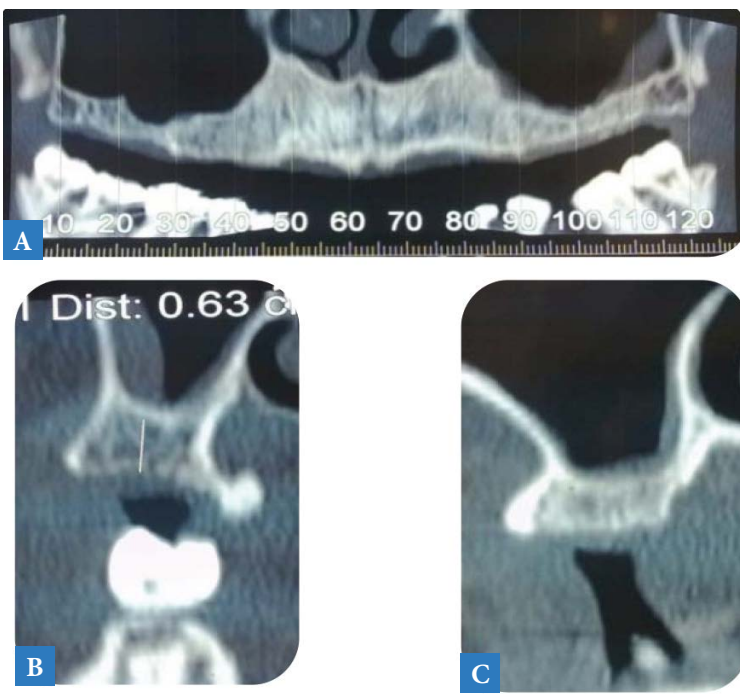

$\triangle$ Fig. 3a, b et $\mathbf{c}$ : Proximité sinusienne et proximité du canal palatin révélées au scanner.

\section{Phase chirurgicale}

La chirurgie correctrice soustractive ne doit pas être mutilante. Elle s'est faite en deux temps. La première étape a concerné le côté droit et la deuxième est réalisée dans une séance ultérieure (une semaine après) et a concerné le côté gauche (Fig. 5a et b).

La chirurgie est guidée par les guides chirurgicaux préparés préalablement à cet effet en respectant les obstacles anatomiques avoisinants à savoir le canal palatin (Fig. 6).

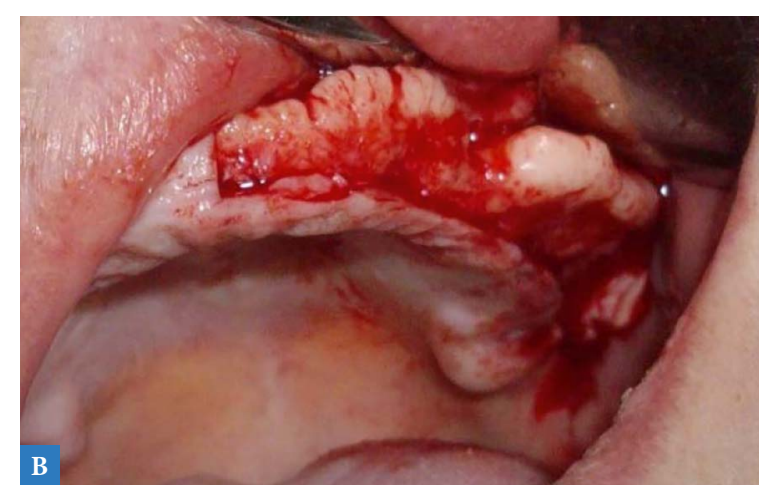

$\triangle$ Fig. 5a et b : Réduction du volume osseux, limitée par le respect du canal palatin. 


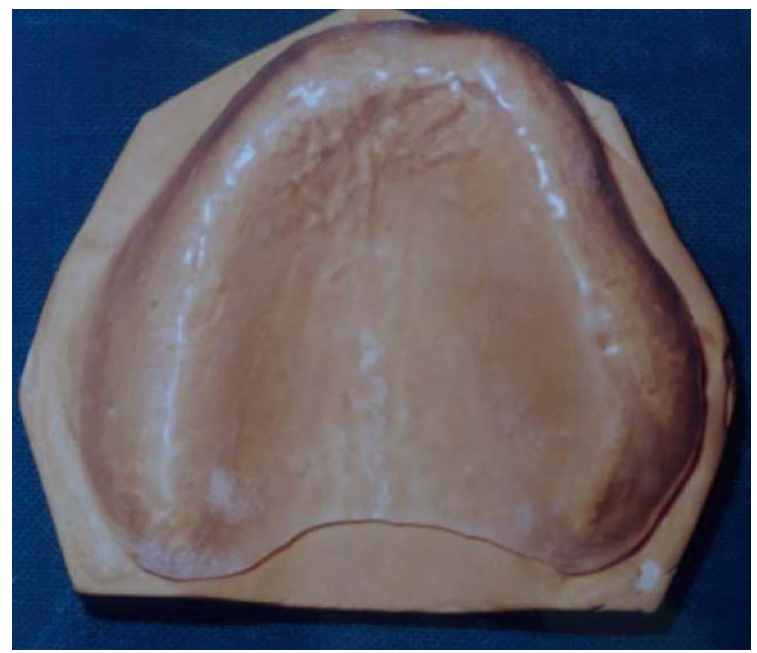

$\triangle$ Fig. 6 : Guide chirurgical sur le modèle maxillaire rectifié.

Au fur et à mesure des soustractions tissulaires, le guide chirurgical est essayé pour vérifier l'importance de la décortication osseuse.

Lors de la $2^{\mathrm{e}}$ phase chirurgicale, un prélèvement osseux est réalisé et destiné à l'examen anatomopathologique révélant ainsi un ostéome.

\section{Mise en place de la prothèse transitoire au maxillaire}

C'est un élément déterminant de la bonne intégration des prothèses définitives car, véritable test clinique et « patron » des réalisations définitives, elle va permettre : $\checkmark$ de guider la cicatrisation grâce à une mise en condition tissulaire ;

$\checkmark$ d'évaluer les déterminants occlusaux mis en place (dimension verticale, plan d'occlusion, relation centrée), les fonctions de mastication, de phonation et l'esthétique ;

Dans notre cas, la prothèse transitoire maxillaire prend l'expression, d'une prothèse immédiate, insérée à l'issue des interventions chirurgicales (Fig. 7a). La difficulté a été de concevoir une prothèse unimaxillaire fonctionnelle et acceptable esthétiquement sans possibilité d'essai clinique. (Fig. 7b).

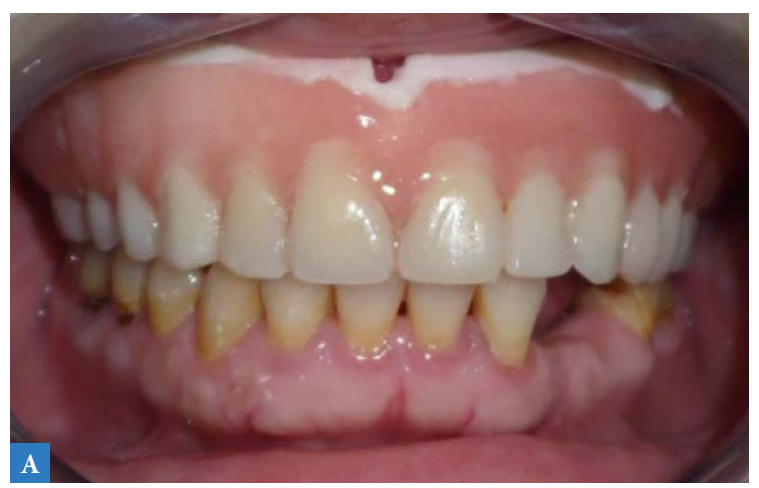

$\triangle$ Fig. 7a : Prothèse immédiate transitoire.

\section{Phase de temporisation}

Les prothèses transitoires immédiates constituent un réel pansement qui protège les sites opératoires. Elles favorisent la cicatrisation en guidant le remodelage des tissus ostéo-muqueux et en minimisant la résorption osseuse grâce à l'application précoce de stimuli fonctionnels. Cette phase dure de 3 à 6 mois avant de passer à la réalisation des prothèses complètes d'usage. La prothèse transitoire est garnie d'une résine à prise retardée : Fitt de Kerr, pour guider la cicatrisation osseuse. Durant la phase de temporisation, le conditionneur est régulièrement renouvelé jusqu'à l'obtention d'une base osseuse stable.

\section{Réalisation de la prothèse d'usage}

Après cicatrisation (Fig. 8), la réalisation de la prothèse d'usage est entamée. Elle est la reproduction fidèle de la prothèse transitoire dont les paramètres fonctionnels et esthétiques ont été testés et validés.

La réhabilitation prothétique de la patiente consiste en la réalisation d'une prothèse amovible complète maxillaire.

L'empreinte anatomo-fonctionnelle du maxillaire est effectuée sous pression digitale; l'empreinte secondaire sous pression occlusale est à proscrire, car le réflexe de propulsion est inhérent à la persistance $d u$ bloc incisivo-canin, le sujet recherchant d'instinct la proprioception au niveau des dents restantes.

Les moulages sont transférés sur articulateur en relation centrée par le biais de maquettes d'occlusion à une dimension verticale d'occlusion correcte.

Le montage des dents prothétiques est fait en adoptant le concept d'occlusion "intégralement équilibrée». Il est essayé et validé en bouche avant de passer à la polymérisation et à la pose de la prothèse. (Fig. 9). La patiente est revue régulièrement afin de contrôler l'absence de contact entre les dents antérieures naturelles et prothétiques et, en cas de contact, l'éliminer. Ainsi, La stabilité et le confort prothétiques sont assurés favorisant l'intégration fonctionnelle de la prothèse et la préservation de l'intégrité tissulaire.

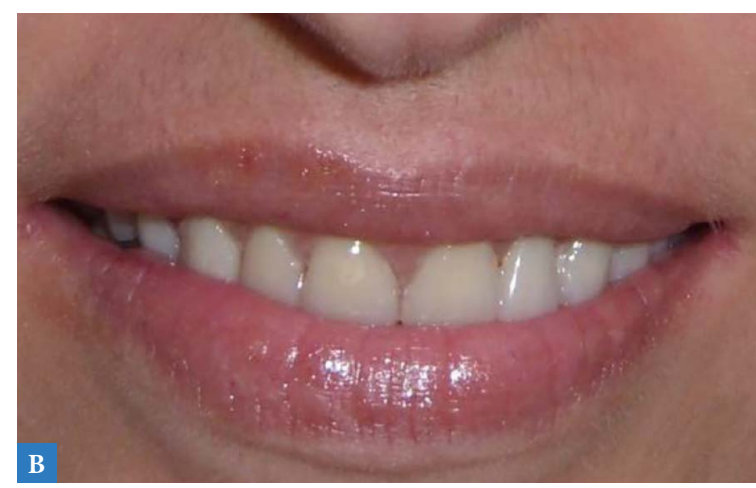

$\triangle$ Fig. $7 \mathbf{b}$ : Sourire de satisfaction de la patiente. 


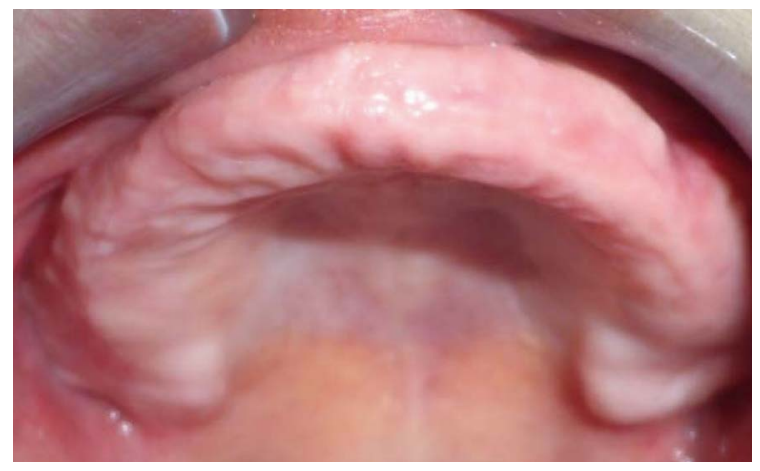

$\triangle$ Fig. 8 : Cicatrisation bien guidée par les prothèses transitoires.

\section{Discussion}

Il existe une grande différence des prévalences et distribution des hypertrophies osseuses buccales entre les différentes études en fonction de la population et du type de l'hypertrophie étudiée.

Antoniades et al. ont étudié un échantillon de 416 dents sélectionnées dans 52 spécimens de squelettes humains modernes au musée national de l'histoire naturelle des États Unies. Les hypertrophies de l'os alvéolaire ont été trouvées dans $25 \%$ de toutes les dents examinées : $18 \%$ ont été exprimées sous forme d'hypertrophies osseuses linéaires marginales dans les régions molaire, prémolaire et incisive mandibulaire et $7 \%$ sous forme d'exostoses buccales [7]. Une étude de Rezai et al. a été basée sur l'observation de 732 crânes, d'origine ethnique diverse, appartenant à la collection d'ostéologie de l'institut d'anatomie normale de Strasbourg. Sur les 732 crânes examinés, 76 présentent un torus palatin, soit $10,5 \%$. Une fréquence relativement importante du torus palatin est cependant retrouvée dans la population d'origine rhénane (Allemagne). Les pourcentages les plus faibles ont été trouvés chez les populations du nord Afrique (Égyptiens anciens et tunisiens). [4]

Une autre étude d'Aree J. et al. [8]. visait la prévalence des exostoses buccales et le rapport avec les tori palatin et mandibulaire chez des patients thaillandais. Sur les 960 sujets étudiés, $26,9 \%$ présentaient des exostoses. Les exostoses étaient plus fréquentes au maxillaire qu'à la mandibule avec un ratio 5,1 : 1. 3,5\% des individus présentaient les deux localisations. Les hommes présentaient plus d'exostoses $(62,4 \%)$ que les femmes (37,6 \%). 90,7 \% des patients avec des exostoses présentaient aussi des tori. Ce qui signifie une forte relation entre exostoses buccales et tori.

L'étiologie des exostoses demeure inconnue, différentes hypothèses peuvent cependant justifier la cause de la forme des os :

Intoxication métallique : à concentrations élevées, certains éléments, notamment le strontium et le fluor, peuvent avoir des effets bénéfiques sur le sque-

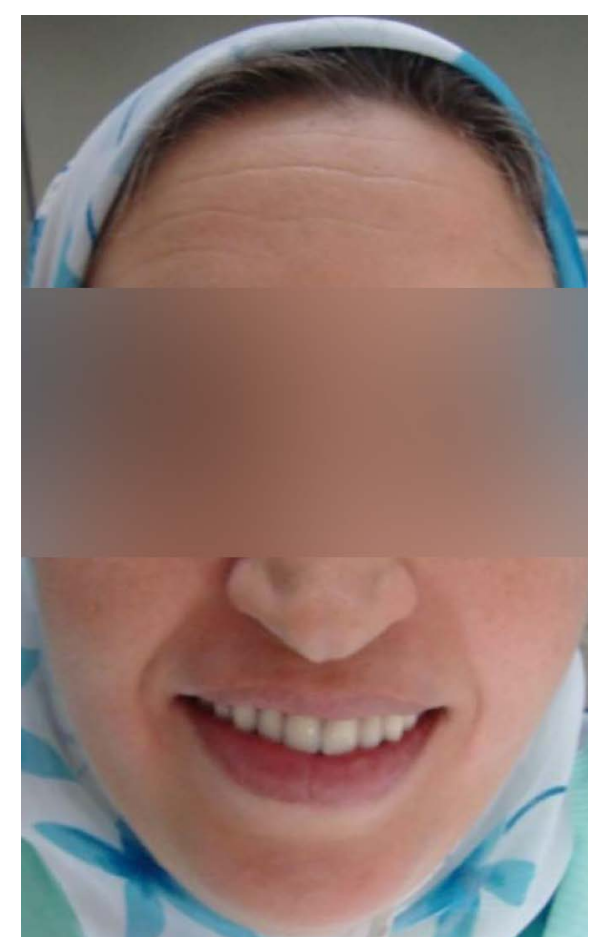

$\triangle$ Fig. 9 : Prothèse définitive maxillaire en bouche.

lette. Toutefois, de plus fortes concentrations sont toxiques. [9].

Fluorose osseuse : les anomalies dentaires s'observent pour des concentrations hydriques en fluorures supérieures à $2 \mathrm{mg} / \mathrm{l}$, alors que les lésions osseuses sont notées à partir d'un taux variant entre 4 et $8 \mathrm{mg} / \mathrm{l}$. [10]

Médication : les hyperostoses constituent un des effets indésirables rares des rétinoïdes. Elles sont constatées après médication à fortes doses et en traitement prolongé. Les rétinoïdes accusés par leur effet hyperostosant sont : l'acitrétine, l'alprostadil, l'étrétinate et l'isotrétinoine.

L'utilisation prolongée de l'hormone de croissance peut également provoquer des hypertrophies osseuses. Celles-ci se caractérisent par une déformation irréversible du visage et de la tête avec un avancement prononcé de la mâchoire inférieure (signe de l'acromégalie) et un allongement des maxillaires. [11]

Stress occlusal : une étude réalisée aux État Unis en 2002 par le département d'anthropologie de l'université Missouri-Columbia sur le rôle du stress occlusal dans la genèse des hypertrophies localisées (exostoses). Les auteurs de cette étude ont signalé une forte corrélation entre des manifestations du stress occlusal (pathologie de l'ATM, usure des surfaces occlusales des dents) et l'apparition des exostoses en particulier les exostoses vestibulaires au niveau maxillaire et linguales à la mandibule. Une deuxième constatation concerne la fréquence des exostoses observée sur les crêtes antérieures des mâchoires édentées postérieurement. Cela est expliqué par le fait que l'absence des 
molaires fait déplacer les charges de mastication vers les dents antérieures. [12]

Il a été démontré expérimentalement que les forces exercées sur les molaires sont redistribuées sur les tissus environnants pour induire le remodelage osseux (Waldo et Rothblatt 1954). [13]

D'autres études sur le bruxisme ont rapporté une corrélation significative avec les exostoses vestibulaires, ce qui est en faveur du rôle des forces occlusales dans la formation des hypertrophies osseuses localisées. [14] Jozefowicz W. a reporté un cas d'hypertrophie importante de l'os alvéolaire de la mandibule, chez un sportif, suite au port de poids lourd avec les dents pendant plusieurs années. [5]

Traumatisme : le traumatisme du périoste semble être l'agent étiologique principal associé au développement d'exostoses buccales dans les zones ou une greffe gingivale libre autogène a été mise. [15] [16]

D'autre part, il a été rapporté des cas d'hyperplasie osseuse subpontic, au niveau des régions molaires. C'est une affection bénigne qui prolifère dans l'os alvéolaire en dessous de l'intermédiaire d'une prothèse fixée. [17]

Infection : l'infection gingivale prolongée a été proposée comme un facteur mineur de la formation d'exostoses. Ultérieurement, une minéralisation du foyer de l'inflammation aboutirait à la formation d'exostose. [3] Prédisposition génétique : il a été remarqué dans de nombreuses observations, que la fréquence, la morphologie et l'extension des exostoses sont calquées sur les populations et persistent dans les familles. Cela suggère la nature génétique du trait. [3]

Grosky et al. estiment quant à eux, que l'étiologie de ces excroissances osseuses est probablement multi-factorielle, incluant des facteurs environnementaux sous une action combinée et complexe de facteurs génétiques. [8] Troubles endocrinologiques : les principales pathologies endocriniennes susceptibles d'entraîner un trouble de la minéralisation sont : l'hyperparathyroïdisme primitif ou secondaire, l'hyperthyroïdie, l'hypercorticisme endo- ou exogène et l'acromégalie. [18] En fonction de l'étiologie de l'hypertrophie osseuse, la prise en charge diffère : ainsi le traitement des hypertrophies osseuses tumorales et kystiques dépendra de la malignité ou de la bénignité de la lésion, de la localisation, de la sévérité, du caractère primitif ou secondaire de la tumeur et de l'histoire de la lésion (récidive). [19] [20]

Pour les hypertrophies osseuses buccales dont l'origine est une pathologie générale, le traitement ne concernera que les cas symptomatiques et/ou compliqués ou les cas d'hypertrophies qui interfèrent avec la mise en place d'une prothèse adjointe stable. Dans ce cas, à côté du traitement médical prescrit par le médecin spécialisé, le traitement de l'hypertrophie osseuse buccale consistera en une chirurgie remodelante. [21] De même, pour les hypertrophies osseuses buccales, d'origine locale ou toxique, et qui interfèrent avec la réalisation de la prothèse adjointe, seront éliminées par chirurgie remodelante. [22] [23]

Si le patient est inopérable ou présente une contre indication de la chirurgie et si le volume de l'hypertrophie osseuse le permet, on optera pour une prothèse déchargée sans intervention chirurgicale pour l'édentement total.

\section{CONCLUSION}

Les hypertrophies osseuses des maxillaires sont rares. Mais, quand elles existent, elles peuvent constituer un véritable obstacle pour la réhabilitation prothétique chez l'édenté.

La prise en charge des patients présentant ces hyperostoses, nécessite la détermination de leurs étiologies pour les prendre en considération dans la décision thérapeutique, et dans le suivi du patient.

Souvent, quand l'état du patient le permet, la chirurgie osseuse soustractive est de recours.

Mais si le patient présente une ou plusieurs contre-indications de la chirurgie, on essayera de réaliser une prothèse amovible qui contourne la proéminence osseuse.

Vu la diversité des facteurs étiologiques de ces hypertrophies, la prise en charge se trouve complexée d'autant plus que dans certains cas, l'hypertrophie osseuse récidive après la chirurgie. Mais, la chirurgie préprothétique donne des résultats satisfaisants du point de vue fonctionnel, esthétique et relationnel pour le patient. 


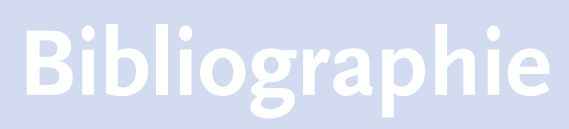

[1] Castany E, Laffargue P et Godet C : Aménagement pré-prothétique des crêtes édentées: six situations cliniques fréquentes. Inf Dent 2002; 38: 2868-2874.

[2] Sefrioui A, Berrada S: Aménagement de l'espace prothétique en prothèse amovible complète (à propos d'un cas clinique). Actual Odonto-Stomatol 2005; 230: 123-136.

[3] Horning GM, Cohen ME, Neils TA: Buccal alveolar exostoses: prevalence, characteristics, and evidence for buttressing bone. J Periodontol 2000; 71 (6): 1032-42.

[4] Rezai RF, Jackson JT, Salamat K: Torus palatinus, an exostosis of unknown etiology: review of the literature. Compend Contin Educ Dent 1985; 6:149-152.

[5] Jozefowicz W: A case of considerable proliferation of the alveolar part of the shaft of the mandible as a result of lifting weights by means of teeth lasting for many years. Czas Stomatol 1965; 18 (7): 819-821.

[6] Auriol M-M, Le Charpentier Y : Histologie de la muqueuse buccale et des maxillaires. Encycl. Méd Chir, Odontologie, 22-007-M-10, 1998; Médecine Buccale, 28-120-M-10, 2008

[7] Antoniades DZ, Belazi M, Papanayiotou P : Concurrence of torus palatinus with palatal and buccal exostoses. Case report and review of the literature. Oral Surg Oral Med Oral Pathol Oral Radiol Endod 1998; 85: 552-7.

[8] Aree J et al.: Buccal and palatal exostoses: prevalence and concurrence with tori. Oral Surgery Oral Medecine Oral Pathology 2000; 90 (1): 48-53.

[9] Boivin G, Cournot G : Manifestations osseuses et articulaires des intoxications métalliques et métallö̈diques. Encycl. Méd Chir, Appareil locomoteur, 14-025-R-10, 2001.

[10] Laatar A, Mrabet D, Zarkaoui L : La fluorose en Afrique subsaharienne. Rev Rhumatisme 2003; (70) : 178-182.

[11] Javier RM, Sibilia J, Durckel J et Kuntz JL: Ostéopathies médicamenteuses. J Radiol 1999; 80: 709-713.

[12] Pechenkina EA, Benfer Ra JR: The role of occlusal stress and gingival infection in the formation of exostoses on mandible and maxilla from Neolithic China. Homo 2002; 53 (2):112-130.

[13] Krishnan V, Davidovitch Z: Biological mechanisms of tooth movement. John Wiley \& Sons édit. New Jersey, 2009.

[14] Rothblatt JM, Waldo CM: Histologic response to tooth movement in the laboratory rat: procedure and preliminary observations. J Dent Res 1954; 33: 481-486.

[15] Chambrone LA, Chambrone L: Bony exostoses developed subsequent to free gingival grafts: case series. Br Dent J 2005; 199 (3): 146-149.

[16] Colombier M-L, Lesclous P, Tulasne J-F : La cicatrisation des greffes osseuses. Rev
Stomatol Chir Maxillofac 2005; 106 (3): 157-165.

[17 Kevin B et al.: A case of subpontic osseous hyperplasia in the maxillary arch. Oral Surg Oral Med Oral Path Oral Radiol Endod 2000; 89: 73-76.

[18] Alexandre C: Maladie osseuse de Paget. Encycl. Méd Chir, Appareil Locomoteur, 14-023R-10, 2006.

[19] Ruhin B, Guilbert F et Bertrand J-C: Traitement des kystes, tumeurs et pseudotumeurs bénignes des maxillaires. Encycl. Méd Chir, Stomatologie, 22-062-K-10, 2005, Médecine Buccale, 28-550-V-10, 2008.

[20] Barthelemy I, Sannajust J-P, Revol P et Mondie J-M : Cancers de la cavité buccale. Préambule, épidémiologie, étude clinique. Encycl. Méd Chir, Stomatologie, 22-063-A-10, 2005; Médecine Buccale, 28-555-G-10, 2008.

[21] Mariani P, Méyère P: Hypertrophies tubérositaires et prothèse adjointe complète. Obstacle majeur dans la thérapeutique de l'édentement total. Cah Proth 1994; 85: 20- 28.

[22] Princ G, Toledo R, Dichamp J. : Chirurgie préprothétique. Encycl. Méd Chir, Stomatologie, 22-320-A-10, 1999.

[23] Fajri L, Berrada S, Abdedine A. : L'apport de l'exploration clinique dans le choix et l'orientation de la thérapeutique prothétique chez l'édenté complet. ROS 2008; 37 (2): 91-107. 